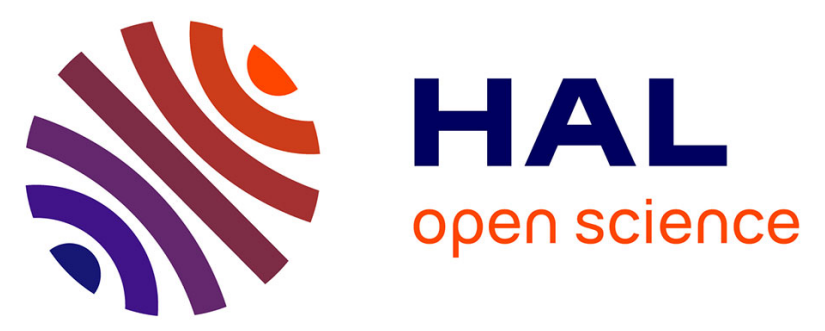

\title{
2D particle-in-cell simulations of the electron drift instability and associated anomalous electron transport in Hall-effect thrusters
}

Vivien Croes, Trevor Lafleur, Zdeněk Bonaventura, Anne Bourdon, Pascal Chabert

\section{To cite this version:}

Vivien Croes, Trevor Lafleur, Zdeněk Bonaventura, Anne Bourdon, Pascal Chabert. 2D particlein-cell simulations of the electron drift instability and associated anomalous electron transport in Hall-effect thrusters. Plasma Sources Science and Technology, 2017, 26 (3), pp.034001 10.1088/13616595/aa550f . hal-01510374

\section{HAL Id: hal-01510374 https://hal.sorbonne-universite.fr/hal-01510374}

Submitted on 19 Apr 2017

HAL is a multi-disciplinary open access archive for the deposit and dissemination of scientific research documents, whether they are published or not. The documents may come from teaching and research institutions in France or abroad, or from public or private research centers.
L'archive ouverte pluridisciplinaire HAL, est destinée au dépôt et à la diffusion de documents scientifiques de niveau recherche, publiés ou non, émanant des établissements d'enseignement et de recherche français ou étrangers, des laboratoires publics ou privés. 


\title{
2D Particle-In-Cell simulations of the electron drift instability and associated anomalous electron transport in Hall-Effect Thrusters
}

\author{
Vivien Croes $^{1,2} \ddagger$, Trevor Lafleur ${ }^{1,3}$, Zdeněk Bonaventura ${ }^{4}$, Anne \\ Bourdon $^{1}$, Pascal Chabert ${ }^{1}$ \\ ${ }^{1}$ Laboratoire de Physique des Plasmas, CNRS, Sorbonne Universités, UPMC \\ Université Paris 6, Université Paris Sud, École Polytechnique, F-91120 Palaiseau, \\ France \\ 2 Safran Aircraft Engines, Electric Propulsion Unit, F-27208 Vernon, France \\ 3 Centre National d'Études Spatiales (CNES), F-31401 Toulouse, France \\ 4 Department of Physical Electronics, Faculty of Science, Masarykova Univerzita, \\ CZ-13485 Brno, Czech Republic
}

November 2016

\begin{abstract}
In this work we study the electron drift instability in Hall-effect thrusters (HETs) using a 2D electrostatic particle-in-cell (PIC) simulation. The simulation is configured with a Cartesian coordinate system modeling the radial-azimuthal $(r-\theta)$ plane for large radius thrusters. A magnetic field, $\mathbf{B}_{0}$, is aligned along the $O y$ axis $(r$ direction), a constant applied electric field, $\mathbf{E}_{0}$, along the $O z$ axis (perpendicular to the simulation plane), and the $\mathbf{E}_{0} \times \mathbf{B}_{0}$ direction is along the $O x$ axis ( $\theta$ direction). Although electron transport can be well described by electron-neutral collisions for low plasma densities, at high densities (similar to those in typical HETs), a strong instability is observed that enhances the electron cross-field mobility; even in the absence of electron-neutral collisions. The instability generates high frequency (of the order of $\mathrm{MHz}$ ) and short wavelength (of the order of $\mathrm{mm}$ ) fluctuations in both the azimuthal electric field and charged particle densities, and propagates in the $\mathbf{E}_{0} \times \mathbf{B}_{0}$ direction with a velocity close to the ion sound speed. The correlation between the electric field and density fluctuations (which leads to an enhanced electron-ion friction force) is investigated and shown to be directly responsible for the increased electron transport. Results are compared with a recent kinetic theory, showing good agreement with the instability properties and electron transport.
\end{abstract}

Keywords: Hall Effect Thruster (HET), 2D Particle-In-Cell (PIC) simulation, Anomalous electron transport, Electron drift instability

Submitted to: Plasma Sources Sci. Technol.

† Email: vivien.croes@lpp.polytechnique.fr 


\section{Introduction}

Hall-effect thrusters (HETs) are one of the most successful technologies used to electrically generate thrust for satellites. The first in-space demonstration of electric propulsion (EP) was made by an ion engine in 1964 aboard the SERT-1 spacecraft [1], followed by the first HET in 1974 aboard the USSR Meteor satellite [2]. Since then numerous satellites have been successfully operated using HETs as primary or secondary propulsion systems, for commercial, military or exploration purposes [3]. However the full potential of EP, and in particular HETs, has only started to be realized in the last few years with the appearance of all-electric communication satellites, and large small-satellite constellation projects [4].

Typical HETs used on these spacecraft consist of three main parts as shown in Figure 1: (1) An annular ceramic channel where the propellant gas is injected (through a porous anode), ionized, accelerated, and ejected through one end. This channel usually has a length of the order of centimeters [5]. In addition, densities in the channel are typically in the range between $10^{17}$ to $10^{18} \mathrm{~m}^{-3}$ for the plasma, and $10^{18}$ to $10^{20} \mathrm{~m}^{-3}$ for the neutral gas [6]. (2) An external hollow cathode (asymmetrically located near one side of the channel, or along its central axis), providing electrons to sustain the plasma discharge inside the channel, as well as neutralizing the exiting ion beam. A large potential difference (100's of Volts) is applied between the anode and cathode, which accelerates the ions to high velocities to generate thrust. This potential difference also causes some electrons from the cathode to travel upstream inside the channel towards the anode. (3) A specially designed magnetic circuit used to impose a predominantly radial magnetic field (10's of $\mathrm{mT}$ ) in the channel region. This magnetic field acts to impede electrons and increases their residence time in the channel so as to allow a higher probability of ionization, and thus ensure maximal use of the injected propellant gas [3]. Since the applied discharge voltage gives an axial electric field, and the applied magnetic field is predominantly in the radial direction, this causes an $\mathbf{E}_{0} \times \mathbf{B}_{0}$ drift of electrons in the azimuthal direction. For typical electric and magnetic field values, such as those to be used in the subsequent simulations, the electron drift velocity can reach values as high as about $10^{6} \mathrm{~ms}^{-1}$.

Numerous studies have shown that the electron mobility across the magnetic field tends to be anomalously high in comparison to the mobility predicted by classical diffusion theories based on standard electron-neutral or electron-ion collisions $[7,3,8,6]$, particularly near the thruster exit and in the near-plume region $[8,6,9,10]$. Historically four main mechanisms have been proposed to explain this anomaly, including: (1) Intense secondary electron emission arising from electron-wall collisions enhancing the electron mobility near the channel walls $[7,11,12],(2)$ sheath instabilities in the radial direction due to intense secondary electron emission [12, 13], (3) Large electron drift velocities in the azimuthal direction leading to the formation of strong instabilities $[14,15,16,17]$, and (4) gradient driven fluid instabilities [18, 19, 20, 21]. The role of thruster walls materials on this anomalous transport has been experimentally 


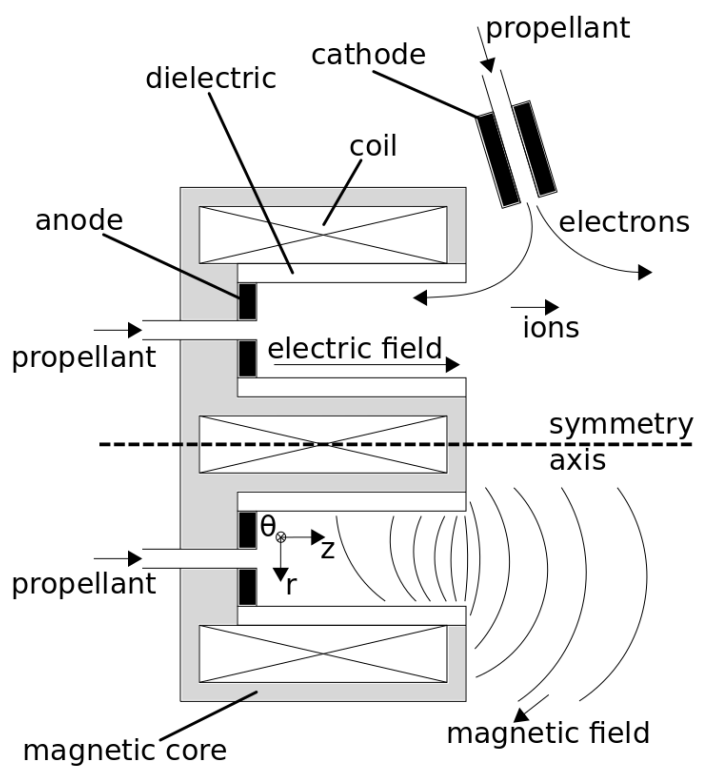

Figure 1: Schematic of a typical HET.

highlighted in numerous studies [3, 11, 12, 22], however evidence suggests that electronwall collisions and secondary electron emission are not sufficient to explain the observed cross-field electron transport [14, 23, 24, 25, 26, 27].

Thus the most plausible explanation seems to be the formation of instabilities in the azimuthal direction, as highlighted by both experimental and numerical studies $[28,29,30,31,32]$. The large electron drift velocity in the azimuthal direction appears to be the main driving force for these instabilities [15, 16, 33], which give large amplitude fluctuations in both the plasma density and azimuthal electric field. The importance of these instabilities on electron transport was highlighted in [15], which modeled a HET in a $2 \mathrm{D} z-\theta$ plane using PIC simulations. These simulations were able to reproduce self-consistently numerous experimental observations, without requiring the addition of any empirical parameters to increase the electron collisionality. Although the channel walls or secondary electron emission were not explicitly modeled, these simulations observed a strong electron drift instability in the azimuthal direction, suggesting this as the main phenomenon causing the anomalous electron transport [6]. These instabilities have frequencies in the $\mathrm{MHz}$ range, wavelengths of the order of the $\mathrm{mm}$, and electric field amplitudes almost as large as the axial accelerating field itself [6]. Some of these results were independently confirmed by similar 2D simulations [34].

Complementing these 2D studies, recent 1D PIC simulations have been developed to more rapidly gain insight into the electron drift instability and resulting electron transport. As proposed in [35], and independently verified in [36], it is possible to model the instability in a 1D system by simulating the azimuthal direction (where Poisson's equation is solved) and including a finite axial length (where Poisson's equation is not solved, but a fixed electric field is applied). This finite axial length is needed in order to model convection of the instability and to ensure saturation. Otherwise, as shown in 
previous 2D PIC models [15], simulations do not reach a steady-state and the electron energy continues to increase in time, meaning that only about $1-2 \mu$ s of the discharge can be simulated. Using 1D PIC models with a finite axial length, it is possible to observe anomalous electron transport and uncover important insights from the instability, since now a steady-state can be reached. As a result of these insights, a kinetic theory was proposed to theoretically explain the anomalous electron transport [37]. This theoretical work shows that the electron drift instability leads to an enhanced electron-ion friction force. This force results from the correlation between oscillations in the electron density and azimuthal electric field, leading to an enhanced electron cross-field mobility.

In this paper we develop a 2D PIC simulation to extend previous 1D studies by using a more realistic geometry that includes the radial thruster walls. The main objective of this work is to investigate the importance of $2 \mathrm{D}$ effects, and to compare the results with both the previous 1D PIC simulations in [35] and [36], and with the recent kinetic theory in [37]. Even in 2D, PIC simulations can be very time consuming, typically requiring of the order of weeks or even months to run depending on the operating conditions and system geometry. Scaling methods, such as reducing the electron density or increasing the permittivity of space, can be used to reduce computational times, however these scalings appear to directly affect the instability growth rate, amplitude, and hence the electron transport. Therefore in this work an effort was made to develop a parallelized code to benefit from developments in high performance computing.

\section{Model Presentation}

The results presented in this article were obtained using a 2D-3V Particle-in-cell (PIC) code: LPPic2D, independently developed. In this code, a simple geometry is used, without any scaling methods, so as to preserve all spatial and temporal scales.

\subsection{Particle-In-Cell (PIC) simulations}

The LPPic2D code uses the classical structure of a 2D PIC code, as described in [38]. This simulation uses a Cartesian structured mesh, fixed in time, with square cells $(\Delta x=\Delta y)$. For each loop the system models a time-step, $\Delta t$, of physical time. This time-step is a parameter in the simulation and is chosen so as to resolve the electron plasma frequency, and to satisfy the Courant-Friedrichs-Lewy (CFL) condition.

The LPPic2D simulation code uses the MPI library to parallelize the calculations using a classical 2D spatial domain decomposition method [39]. The code uses the leapfrog scheme for both charged species (ions and electrons) to explicitly integrate the equations of motion. In the case where a magnetic field is present, the Boris scheme [40] is used to obtain the electron trajectories (ions are assumed to be unmagnetized). Neutrals are not followed in the simulation, but are treated as a fixed, homogeneous, background at a given temperature, $T_{n}$, and pressure, $P_{n}$. Collisional processes between charged particles and the neutral background are modeled using the Monte Carlo 
Collision (MCC) algorithm described in [41]. Ionization processes, as well as excitation and elastic collision processes, are modeled for electrons, while charge-exchange and elastic collision processes are modeled for ions. The simulation code is designed to model either a Xenon plasma or a Helium plasma (in order to complete the benchmark verification tests described in the Appendix), with cross-sections obtained from [42] and [43] respectively. The charged particle densities at the grid points (and electric fields at the particle positions) are obtained using a standard 2D linear weighting scheme [38]. Since the simulation is electrostatic, the electric field is obtained from the plasma potential by solving Poisson's equation on the grid points. This is done using a finite difference method [38], and the open source HYPRE matrix solver library [44]. Finally, diagnostics are used to store relevant simulation outputs. In order to reduce the level of statistical noise in the simulations, results are averaged over a certain number of time-steps, $N_{A}$. The storage of data is then done using the HDF5 library [45]. With the code developed in this work, the HET simulations presented in Section 2.2 use about 100 particles per cell with $2.5 \times 10^{5}$ grid points, and require less than a week on 63 CPUs to simulate $2.5 \times 10^{6}$ time steps (about $10 \mu \mathrm{s}$ ).

Despite being numerically simple, the complexity of such a program, requires numerous testing and benchmarking processes, which we discuss in the Appendix.

\subsection{D infinite thruster model}

The LPPic2D code has been used to model a system close to a HET thruster, in particular, simulating the $r-\theta$ plane. Because HETs are complex devices, we have chosen a simplified model that does not include any curvature or secondary electron emission, and where the radial walls are metallic, not insulating, and grounded. These omissions are clearly an oversimplification of a real thruster, but they allow us to more clearly focus on the direct effects of the electron drift instability itself. We consider a HET with an infinite radius (so that curvature effects are not important), and model a thin slice of the thruster channel in the $r-\theta$ direction. In this case the simulation domain is a rectangle, closed by walls in the $r$ direction and periodic in the $\theta$ direction (Figure $2)$. Thus we can now use a Cartesian coordinate system $(O x, O y, O z)$ corresponding to the cylindrical coordinate system $(\theta, r, z)$ to describe the simulation domain. In order to keep the notations clear and avoid confusion, Cartesian coordinates will be used from now on in the following sections.

In this geometry, a uniform magnetic field, $\mathbf{B}_{0}$, is applied in the $O y$ direction, and a uniform electric field, $\mathbf{E}_{0}$, is applied in the $O z$ direction, perpendicular to the $(O x-O y)$ simulation plane. The $\mathbf{E}_{0} \times \mathbf{B}_{0}$ direction is then along the $O x$ axis. Particle positions and velocities are tracked in all three dimensions, $(O x, O y, O z)$. Although the applied electric field in the $O z$ direction is arbitrarily imposed in the system (and constant in space and time), the electric field in the simulation plane is obtained self-consistently by solving Poisson's equation. To get the charge densities needed to solve Poisson's equation, the particles are all weighted to the same 2D $(O x-O y)$ grid regardless of 
their position along $O z$. Thus we estimate the densities using the projection along $O z$ of each particle on the $(O x-O y)$ simulation plane. Particles are then moved by integrating the equations of motion. Although for electrons a Boris scheme [40] is used to include the magnetic field, since the Larmor radius of ions is much greater than the simulation domain, the effect of the magnetic field on their trajectories is neglected. In this simulation, electrons and singly-charged Xenon ions are modeled. Electron-neutral and ion-neutral collision processes are included using the same MCC algorithm as described previously. Electron-neutral collisions include elastic collisions, four excitation processes $(8.315 \mathrm{eV}, 9.447 \mathrm{eV}, 9.917 \mathrm{eV}$, and $11.7 \mathrm{eV})$, and ionization collisions, while ion-neutral collisions include charge exchange and elastic scattering. Cross-sections are taken from [42]. Coulomb collisions between charged particles are not explicitly modeled in these simulations. During an ionization process, only the energy loss and velocity scattering are modeled and no new ion or electron is added to the simulation. This is done in order to keep the number of particles in the system essentially constant.

The $(O x-O y)$ simulation plane is closed by grounded metallic walls in the $O y$ direction. Particles are absorbed by these walls without any secondary electron emission process. However, in order to keep the number of macro-particles in the system relatively constant at a steady state, some of these absorbed particles have to be re-injected. This is done by tracking the number of ions and electrons having hit the walls during the precedent time-steps, represented as $\mathscr{N}_{\text {ions }}^{\text {absorbed }}(t), \mathscr{N}_{\text {electrons }}^{\text {absorbed }}(t)$. At the end of the time-step, the number of particles to be re-injected, $\mathscr{N}_{\text {couples }}(t)$, is estimated as $\mathscr{N}_{\text {couples }}(t)=\min \left(\mathscr{N}_{\text {ions }}^{\text {absorbed }}(t), \mathscr{N}_{\text {electrons }}^{\text {absorbed }}(t)\right)$. These couples are then uniformly reinjected back into the system. New couples are loaded at the same position with the initial temperatures $\left(T_{e}=5 \mathrm{eV}\right.$ and $\left.T_{i}=0.1 \mathrm{eV}\right)$. In order to take into account unpaired particles that have been absorbed by the walls, we have $\mathscr{N}_{\text {ions }}^{\text {absorbed }}(t+\Delta t)=$ $\mathscr{N}_{\text {ions }}^{\text {absorbed }}(t)-\mathscr{N}_{\text {couples }}(t)+\mathscr{N}^{\text {absorbed }}(t, t+\Delta t)$, with $\mathscr{N}^{\text {absorbed }}(t, t+\Delta t)$ the number of ions having hit the walls between $t$ and $t+\Delta t$. The same process is done with the electrons. These unpaired partners thus allow a charge imbalance to exist in the simulation, and hence for sheaths at the walls to form. This above procedure is similar to that used in the 2D PIC simulations in [15]. As a test, by setting $\mathbf{E}_{0}=\mathbf{B}_{0}=\mathbf{0}$, a sheath forms with a plasma potential of about $5-6 T_{e}$, as expected from standard sheath theory.

In order to solve Poisson's equation in the $(O x-O y)$ simulation plane, periodic conditions are used in the $O x$ direction, while grounded metallic walls are used in the $O y$ direction. Although Poisson's equation is not solved along the $O z$ direction, the simulation domain does have a finite length in this direction for the particles. This is needed because an infinitely long system along $O z\left(L_{z} \mapsto \infty\right)$ would lead to the charged particles constantly being accelerated by the applied electric field, $E_{0}$. Figure 3a shows that for $L_{z} \mapsto \infty$ (without any collisional processes), the mean electronic energy increases to nonphysically high values within about $1 \mu \mathrm{s}$, and no steady-state is reached. To allow the modeled system to reach a steady-state, the system length along 


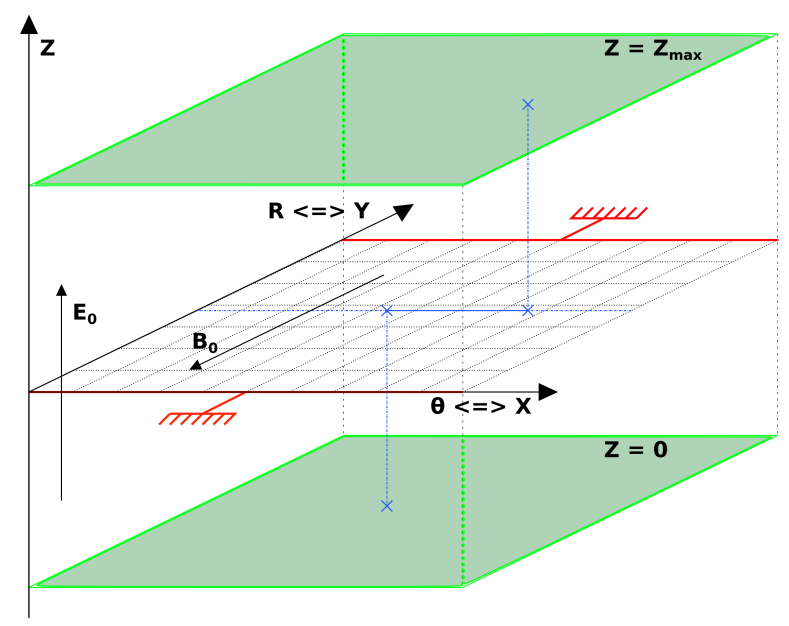

Figure 2: 2.5D capacitive model. Grounded metallic walls (along $O y$ ) close the system (red), periodicity along $O x, L_{z}$ can be finite (green) and then leads to re-injection of charged particles uniformly along $O x$ (blue).

$O z, L_{z}$, is set to a finite value. Thus the simulations could be regarded as $2.5 \mathrm{D}$. The value for the axial length is chosen to be $L_{z}=1 \mathrm{~cm}$ in order to repeat the conditions used in the previous 1D simulations in [36] and [35]. In this case, ions which cross the exit plane of the axial region are replaced by new ions (with an initial temperature of $T_{i}=0.1 \mathrm{eV}$ ) injected at the entrance; while electrons which are lost at the entrance (or exit) plane are replaced by new electrons (at $T_{e}=5 \mathrm{eV}$ ) injected at the exit plane. In this way the number of particles followed by the simulation remains constant. Particles injected in this manner are injected with a random position along $O x$ but keeping the same $O y$ position, otherwise the wall sheaths are prevented from correctly forming (since new particles could be loaded in the sheath). For the same conditions as Figure 3a but with $L_{z}=1.0 \mathrm{~cm}$, Figure $3 \mathrm{~b}$ shows that simulations reach a steady-state within about $1-2 \mu \mathrm{s}$.

Using $N$ as the number of macro-particles and $N G$ as the number of grid points, all simulations use $N / N G \approx 100$ particles per cell, with $N G=2.55 \times 10^{5}$ grid points in the $(O x-O y)$ simulation plane to resolve the Debye length, and with time steps of about $\Delta t=4 \times 10^{-12} \mathrm{~s}$ to ensure resolution of the electron plasma frequency and to satisfy the CFL condition. Unless otherwise stated, standard operating conditions and numerical parameters used in the simulations are listed in Table $1 . T_{i}$ and $T_{e}$ are the initial ion and electron temperatures (and temperatures at which particles are re-injected), $n_{0}$ the initial plasma density, $P_{n}, T_{n}$ and $n_{g}$ the neutral pressure, temperature and neutral density. These values have been chosen to be close to those used in the previous 1D PIC codes [35, 36].

As described for the 1D PIC simulation in [36], using the typical operating conditions in Table 1, the wavelength of the electron drift instability is expected to be about $1.8 \mathrm{~mm}$. Thus $L_{x}$ is chosen to be larger than this. 


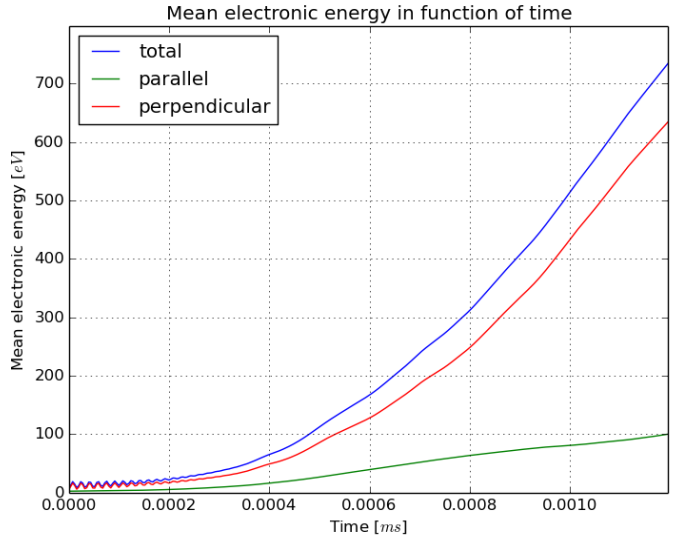

(a)

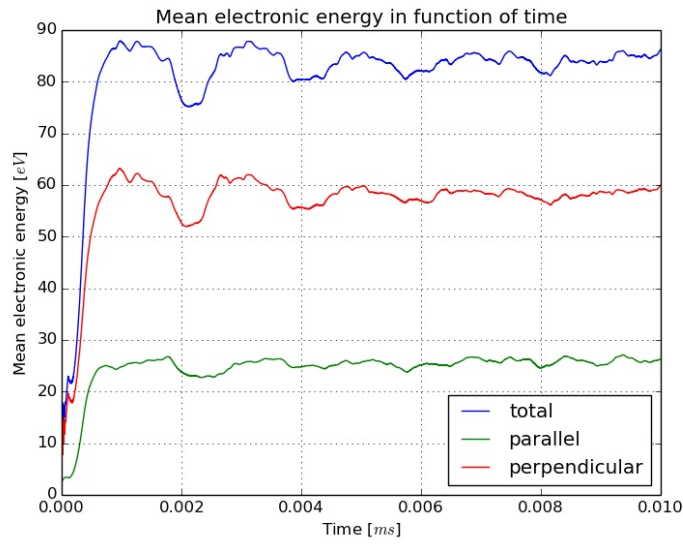

(b)

Figure 3: Time plot of the mean electron energy for (a) Infinite $L_{z}$, (b) $L_{z}=1 \mathrm{~cm}$ : Total energy (blue), parallel to the magnetic field $\mathbf{B}_{0}$ (green), and perpendicular to the magnetic field $\mathbf{B}_{0}$ (red). Both cases are modeled with collision processes turned off.

Table 1: Standard operating and numerical parameters used in the 2D PIC simulations for HET channel.

\begin{tabular}{cc}
\hline Parameter & Value \\
\hline Gas & Xenon \\
$L_{\Theta}[\mathrm{cm}]$ & 0.5 \\
$L_{R}[\mathrm{~cm}]$ & 2.0 \\
$L_{z}[\mathrm{~cm}]$ & 1.0 \\
$B_{0}[\mathrm{G}]$ & 200 \\
$E_{0}\left[\mathrm{Vm}^{-1}\right]$ & $2 \times 10^{4}$ \\
$n_{0}\left[\mathrm{~m}^{-3}\right]$ & $3 \times 10^{17}$ \\
$\Delta t[\mathrm{~s}]$ & $4 \times 10^{-12}$ \\
$\Delta y=\Delta z[\mathrm{~cm}]$ & $2 \times 10^{-5}$ \\
$T_{e}[\mathrm{eV}]$ & 5.0 \\
$T_{i}[\mathrm{eV}]$ & 0.1 \\
$N[$ particles $]$ & $25 \times 10^{6}$ \\
$N G[$ gridpoints $]$ & $255 \times 1000$ \\
$N / N G[$ part $/ \mathrm{cell}]$ & $\approx 100$ \\
$N_{A}[$ time-step $]$ & 2000 \\
$P_{n}\left[\mathrm{~m}_{\text {Torr }}\right]$ & 1.0 \\
$T_{n}[\mathrm{~K}]$ & 300 \\
$n_{g}\left[\mathrm{~m}{ }^{-3}\right]$ & $3.22 \times 10^{19}$ \\
\hline
\end{tabular}

\section{Instability formation}

As shown in Figure $3 \mathrm{~b}$, the discharge requires about $1-2 \mu$ s before the instability saturates and a quasi steady-state is reached, which is characterized by large amplitude 


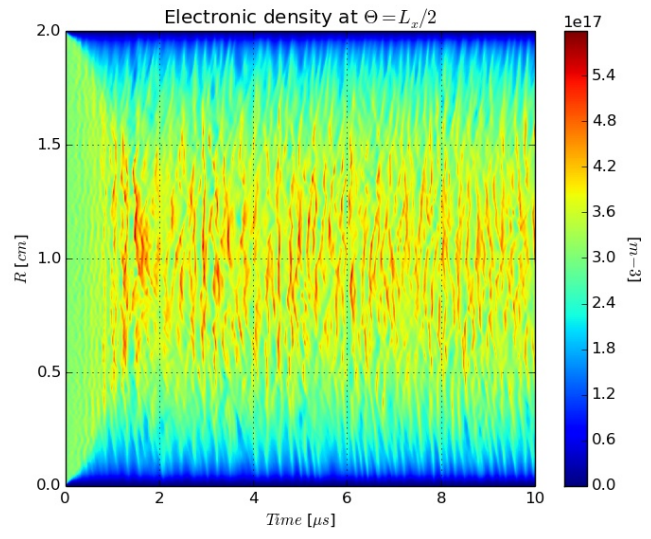

(a)

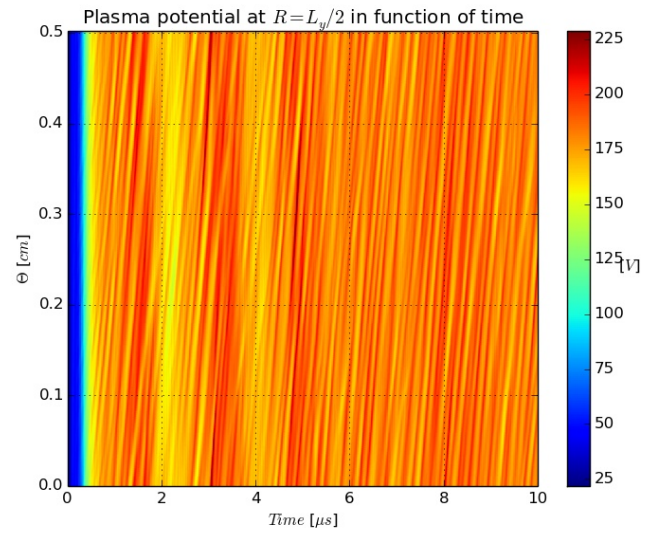

(b)

Figure 4: Plots of the time evolution taken from 1D cuts in the 2D $(O x-O y)$ domain in a non-collisional simulation with parameters from Table 1. (a) Time evolution of the electron density taken along $O y$ (or $r$ ), at $x=L_{x} / 2$ (or $\theta=L_{\theta} / 2$ ), (b) Time evolution of the plasma potential profile taken along $O x$ (or $\theta)$, at $y=L_{y} / 2\left(\right.$ or $\left.r=L_{r} / 2\right)$.

fluctuations in the charged particles densities, electric field, plasma potential and electron energy. In order to visualize the instability formation, saturation and propagation, the time evolution of plasma properties taken along $O x$ (resp. $O y$ ) at position $y=L_{y} / 2$ (resp. $x=L_{x} / 2$ ) are plotted. Since the instability is propagating along $O x$, cuts along $O y$ allow us to study the sheaths and any tilt in the instability propagation, while cuts along $O x$ allow the study of the propagation velocity. Such examples can be seen in Figure 4a for the electron density along $O y$, and Figure 4b for the plasma potential taken along $O x$.

The plot of the electron density shows that it takes about $1 \mu s$ before the initially uniform state fully develops the sheaths at the radial walls. Fluctuations in the density are around $20-30 \%$ of the equilibrium value, and there is also a clearly visible spatially and temporally periodic structure that can be seen in both figures, which gives a wavelength of about $1.7 \mathrm{~mm}$, and a frequency around $5 \mathrm{MHz}$. These values are close to those observed in $[15,16,17,34]$.

A second, lower frequency, fluctuation is also observable in Figure 4b, as well as in Figure 3b. This fluctuation has a frequency of approximately $0.5 \mathrm{MHz}$ and seems to be related to the finite length in the $O z$ direction, since it is absent from Figure 3a. Indeed, when collisions are turned off, ions injected at $z=0$ need a certain transit time to reach $z=z_{\max }$. Since the mean ion velocity along $O z$ is approximately $10^{4} \mathrm{~ms}^{-1}$ and $L_{z}=1.0 \mathrm{~cm}$, we can estimate a transit time of $10^{-6} \mathrm{~s}$. This means that most of the ion population is "renewed" with a frequency of $1 \mathrm{MHz}$, which is close to the low frequency fluctuation observed. In order to confirm this observation, we have replaced the constant acceleration of ions by the applied $E_{0}$ field described in Subsection 2.2, by a constant ion velocity along $O z$ (i.e. the electric field for the ions has been turned 


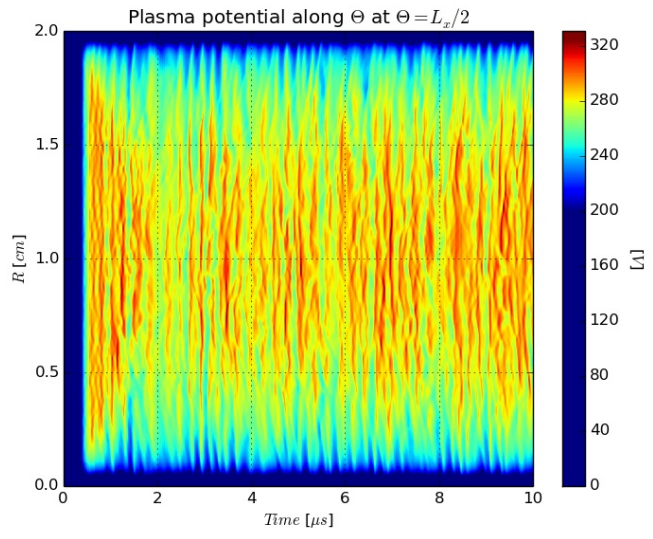

(a)

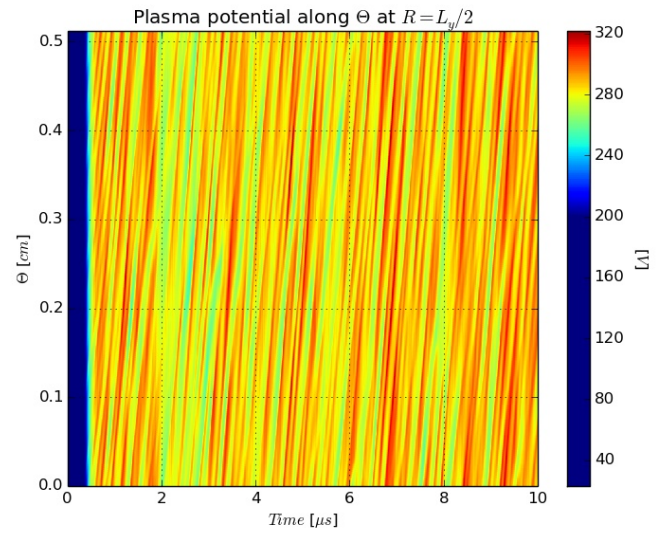

(b)

Figure 5: Time evolution of the plasma potential profile taken from $1 \mathrm{D}$ cuts of the 2D $(O x-O y)$ simulation domain: (a) taken along $O y$ (or $r)$, at $x=L_{x} / 2$ (or $\theta=L_{\theta} / 2$ ), (b) taken along $O x$ (or $\theta$ ), at $y=L_{y} / 2$ ( or $\left.r=L_{r} / 2\right)$. Collisions are "turned on" with parameters from Table 1 . The color-bar has been rescaled in order to highlight the instability behavior.

off and they have been given a constant initial drift). We have checked that, with a constant value of $v_{\text {ions }}^{O z}=10^{4} \mathrm{~ms}^{-1}$, results are similar to those obtained before. With a much higher initial velocity of $v_{\text {ions }}^{O z}=10^{5} \mathrm{~ms}^{-1}$, we have observed (not shown) that the frequency of the low-frequency oscillation is now increased (due to the reduced ion transit time across the axial simulation dimension).

Turning on ion-neutral collisions as well as electron-neutral collisions confirms the above transit time hypothesis. In a collisional simulation, the low frequency oscillations are damped, as seen in Figures $5 \mathrm{a}$ and 5b, in comparison to the non-collisional case. An additional test with only electron-neutral collisions on (i.e. with ion-neutral collisions switched off) shows that the low frequency oscillation is again seen. Thus the effect of ion-neutral collisions is to damp the low-frequency oscillation by causing a more uniform loss of ions in time. Figures $5 \mathrm{a}$ and $5 \mathrm{~b}$ show that the instability is only slightly affected by the presence of collisions, with the main change being that the plasma potential is slightly higher with collisions.

As described for the 1D simulations in $[16,36]$, changes of $L_{z}$ and/or $L_{y}$ (not shown here for the sake of clarity) directly impact the instability behavior, thus $L_{z}$ and $L_{y}$ have to be chosen carefully. More detailed discussion about this is to be found in $[16,36]$.

\section{Ion trapping}

As described in Section 3, the instability needs $\approx 1-2 \mu$ s to saturate and reach a steady-state. After a few growth times of the instability, nonlinear effects begin to set in and limit subsequent growth. This saturation often occurs due to particle-wave 


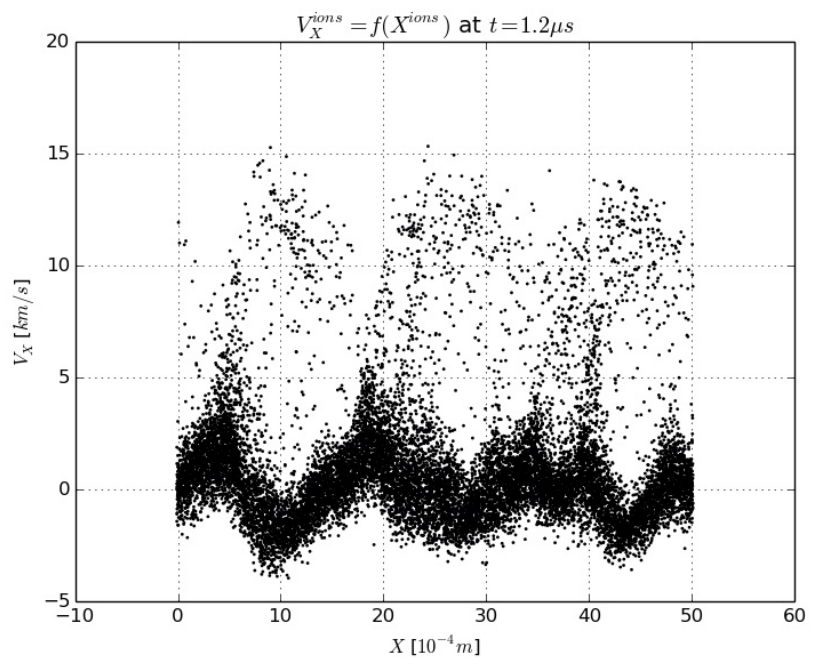

Figure 6: Ion phase space at $t=1.2 \mu s$ in the $(O x)$ direction showing the characteristics "loops" indicative of ion-wave trapping.

trapping [46]. After observation of the electron and ion phase space plots, it is found that the electrons show no obvious signs of trapping. The ions however show strong signs of trapping, as can be seen in Figure 6, which presents a case where collisions were switched off and with simulation parameters equal to those in Table 1. Similar ion trapping was observed in all other simulations where the instability formed.

These results confirm those highlighted previously by the 1D PIC simulations in [36]. Furthermore it highlights two important characteristics of the ion drift velocity in the $O x$ direction: $(1)\left\langle v_{\text {ions }}^{x}\right\rangle \neq 0$, and $(2)\left\langle v_{\text {ions }}^{x}\right\rangle \approx v_{\text {ions }}^{\text {thermal }}$. These last two points can be seen by calculating the average ion velocity in the $O x$ direction from

$$
\left\langle v_{\text {ions }}^{x}\right\rangle=\frac{\sum_{i=1}^{i=\mathscr{N}_{\text {ions }}} v_{i}^{x}}{\mathscr{N}_{\text {ions }}}
$$

where drift velocities of the order of $0.5-1 \mathrm{kms}^{-1}$ are found. These values are similar to those measured experimentally in [47]. In [48] it is shown that the electron drift instability not only leads to an enhanced electron transport, but also to an enhanced ion rotation in the azimuthal direction with a value given by

$$
\left\langle v_{\text {ions }}^{x}\right\rangle \approx \sqrt{\frac{|q| T_{e}}{96 m_{i}}}
$$

where $m_{i}$ is the ion mass, and $T_{e}=2 / 3\left\langle\epsilon_{e}\right\rangle$ is the electron temperature taken from the mean electron energy $\left\langle\epsilon_{e}\right\rangle$. For typical electron temperatures seen in the 2D PIC simulations, Eqn. 2 predicts ion drift velocities of about $650 \mathrm{~ms}^{-1}$; consistent with those found directly from the PIC. 


\section{Enhanced transport}

In these simulations, the cross-field electron mobility is defined as:

$$
\mu_{\text {pic }}=\frac{\sum_{j=1}^{N_{e^{-}}} v_{j z}}{\mathscr{N}_{e^{-}} E_{0}}
$$

where the summation is over all electrons, $\mathscr{N}_{e^{-}}$, in a specific region of the simulation domain.

Before investigating electron transport in a case where an instability is present, we

firstly confirm that we recover the classical cross-field electron mobility, $\mu_{\text {classical }}$, given below, when no instability is present.

$$
\mu_{\text {classical }}=\frac{\frac{|q|}{m_{e} \nu_{m}}}{1+\frac{\omega_{c e}^{2}}{\nu_{m}^{2}}}
$$

Here $\omega_{c e}=|q| B_{0} / m_{e}$ is the electron cyclotron frequency, and $\nu_{m}$ is the electron-neutral collision frequency. As described by Eqn. 4, if $\nu_{m} \mapsto 0$, then $\mu_{\text {classical }} \mapsto 0$. By setting the macro-particle weight in the PIC simulations to a very small number (giving a very low plasma density), no instability forms and the electric field in the $O x$ direction is near zero. With all other simulation parameters corresponding to those listed in Table 1 (except with collisions "switched off"), we obtain a simulation where the instability is not present and the resulting cross-field electron mobility is, as expected, zero.

In the case where the instability forms, the cross-field electron mobility can be described as (see [36]):

$$
\mu_{\mathrm{eff}}=\frac{\frac{|q|}{m_{e} \nu_{m}}}{1+\frac{\omega_{c e}^{2}}{\nu_{m}^{2}}}\left[1-\frac{\omega_{c e}}{\nu_{m}} \frac{\left\langle n_{e} E_{x}\right\rangle}{n_{e} E_{0}}\right]
$$

where $n_{e}$ is the electron density, $E_{0}$ the norm of the axial electric field and $E_{x}$ is the electric field in the $O x$ direction. As we will see, the correlation term $\left\langle n_{e} E_{x}\right\rangle<0$, and thus fluctuations enhance electron mobility. This correlation term represents an electron-ion friction force, which becomes enhanced above the classical value when an instability occurs, as described in Section 6 below. An important property of Eqn. 5 above is: if $\nu_{m} \mapsto 0$, then $\mu_{\mathrm{eff}} \mapsto-\left\langle n_{e} E_{x}\right\rangle / n_{e} E_{0} B_{0}$, where this term is in general not equal to zero.

To confirm such a description a simulation was made using the parameters described in Table 1, but with collisions turned off. In this simulation, we follow in time (the values are averaged every $T=2 \times 10^{3} \Delta t=8 \mathrm{~ns}$ ) the electron mobility as defined in Eqn. 3 for electrons in the whole system and electrons outside the sheaths, and the 


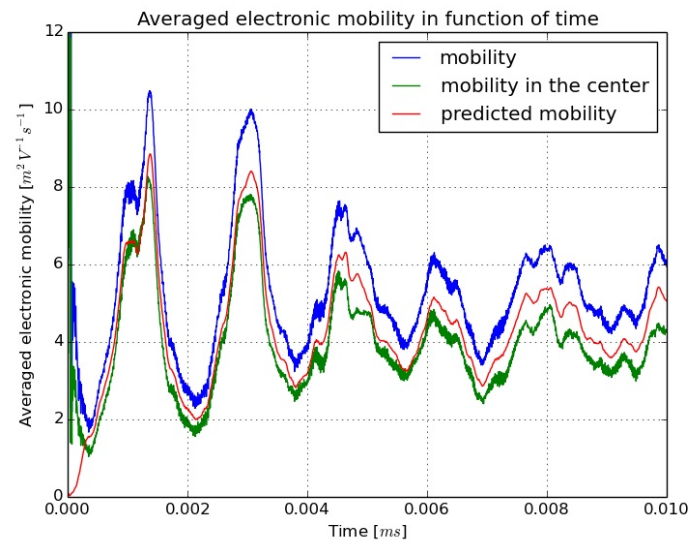

(a)

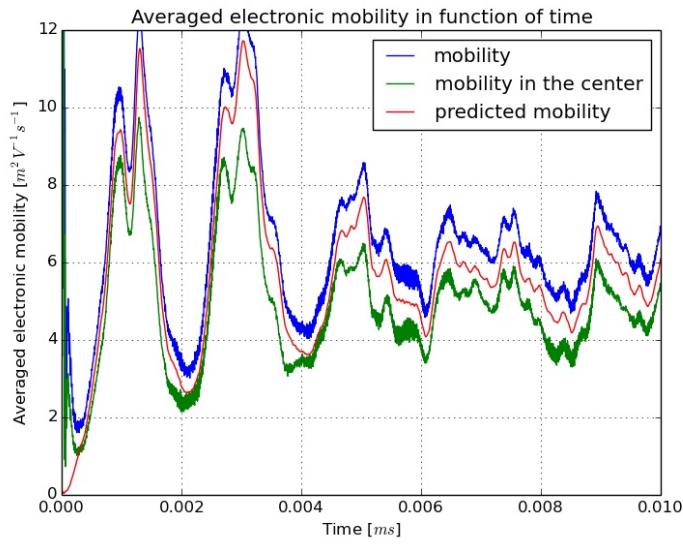

(b)

Figure 7: Time plot of the averaged electron mobility in the system (blue), in the system center (green), and the "predicted mobility", $\mu_{\text {eff }}$, estimated from the correlation term (red), $\left\langle n_{e} E_{x}\right\rangle$ : (a) No collisions, (b) Electron-neutral and ion-neutral collisions enabled.

correlation term $\left\langle n_{e} E_{x}\right\rangle$ using the expression below:

$$
\left\langle n_{e} E_{x}\right\rangle\left(t^{\prime}\right)=\int_{0}^{L_{x}} \frac{d x}{L_{x}} \int_{0}^{L_{y}} \frac{d y}{L_{y}} \int_{t^{\prime}}^{t^{\prime}+T} \frac{d t}{T} n_{e}(x, y, t) E_{x}(x, y, t)
$$

where the integrals are evaluated numerically by using the electron density and electric field along $O x$ given by the simulation at each spatial grid point and at each time-step. From this correlation term we can then estimate $\mu_{\text {eff }}$ thanks to Eqn. 5 with $\nu_{m}=0$. This term $\mu_{\mathrm{eff}}$ is then referred to as "predicted mobility" in the following figures.

The results corresponding to the case $\nu_{m} \mapsto 0$ are shown in the Figure 7a. This figure shows us that: Firstly despite the presence of strong oscillations, the cross-field electron mobility tends to a non-zero value (of about $6.5 \pm 2 \mathrm{~m}^{2} \mathrm{~V}^{-1} \mathrm{~s}^{-1}$ ), as predicted by Eqn. 5, and confirming the 1D PIC results [36]. Secondly, except for the first $0.2 \mu \mathrm{s}$ where the sheaths are forming, $\mu_{\mathrm{eff}}$ seems to be consistent with the measured values of the electron mobility in the system. The very slight difference between the directly computed electron mobility, $\mu_{\text {pic }}($ center $)$, and the "predicted mobility", $\mu_{\text {eff }}$, is most likely due to neglect of the inertial and pressure terms in the electron momentum conservation equation used in the derivation leading to Eqn. 5. Thirdly, the electron mobility in the center of the system (not taking into account electrons in the sheaths, measured by only taking into account electrons between $L_{y} / 4<r<3 L_{y} / 4$ ) is lower than that in the sheaths, since at every time $\mu_{\text {pic }}($ system $)>\mu_{\text {pic }}($ center $)$. Finally we see in Figure 7 that the low frequency oscillation $(\approx 0.5 \mathrm{MHz})$, related to the ion transit time along the $O z$ direction, has an influence on the electron cross-field mobility.

This simulation confirms an important result from the 1D PIC simulations [36]: The enhanced electron transport is essentially independent of electron-neutral collisions. To 
further confirm this result, a simulation is made with $\nu_{m} \neq 0$ and the parameters from Table 1. In this case electron-neutral as well as ion-neutral collisions are modeled, and $\nu_{m}$ is monitored in the simulation using:

$$
\nu_{m}\left(t^{\prime}\right)=\int_{t^{\prime}}^{t^{\prime}+T} \frac{d t}{T} \frac{\mathscr{N}_{\text {collisions }}(t)}{\mathscr{N}_{\text {electrons }}(t)}
$$

Here $\mathscr{N}_{\text {collisions }}(t)$ is the number of electron-neutral collisions at each time-step, and $\mathscr{N}_{\text {electrons }}(t)$ is the number of electrons in the system at each time-step. Although Eqn. 7 is strictly speaking the total collision frequency and not the momentum transfer collision frequency, in the MCC routine we have used isotropic electron scattering, for which it is appropriate to use the momentum transfer scattering cross-sections. Thus in this case, Eqn. 7 correctly yields the momentum transfer collision frequency [49].

The results presented in Figure 7b show that both electron-neutral and ion-neutral collisions at 1 mTorr (which is similar to pressures seen in HETs) are not strong enough to damp the instability, which still continues to be the dominant contribution to the mobility. This agrees well with what was first proposed in [36] and [37].

\section{Comparison with theory}

In order to compare the PIC simulations with the kinetic theory recently developed in [37], we briefly review the main result from this theory, and apply it to the present simulation geometry. The theory in [37] demonstrated that the electron drift instability leads to an enhanced electron-ion friction force, $\mathbf{R}_{e i}$. This force is predominantly in the azimuthal direction, and opposes the electron drift motion with a magnitude given by:

$$
\left|\mathbf{R}_{e i}\right|=\frac{|q|}{4 \sqrt{6}} \frac{1}{c_{s}}\left|\nabla \cdot\left(\mathbf{v}_{d i} n_{e} T_{e}\right)\right|
$$

where $c_{s}=\sqrt{|q| T_{e} / M}$ is the ion sound speed, $\mathbf{v}_{d i}$ is the ion drift velocity, and the derivative, $\nabla$, is over length scales larger than the instability wavelength. In the present PIC simulations ions enter the simulation domain with a very low velocity before being accelerated by the applied axial electric field, $\mathbf{E}_{0}$. Also, there are no large-scale gradients in the radial (except within the thin sheath regions) or azimuthal directions. Thus we can approximate the derivative in Eqn. 8 as: $\nabla \cdot\left(\mathbf{v}_{d i} n_{e} T_{e}\right) \approx \frac{d}{d z}\left(v_{z i} n_{e} T_{e}\right) \approx v_{z i} n_{e} T_{e} / L_{z}$, where $v_{z i}$ is now the final velocity which ions leave the simulation domain with, and $L_{z}$ is the simulation length in the $O z$ direction. Thus Eqn. 8 simplifies to:

$$
\left|\mathbf{R}_{e i}\right| \approx \frac{|q|}{4 \sqrt{6}} \frac{v_{z i} n_{e} T_{e}}{c_{s} L_{z}}
$$

This electron-ion friction force results from the correlation between oscillations in the electron density and electric field, and can more generally be written as $\left|R_{e i}\right|=\left|q\left\langle n_{e} E_{x}\right\rangle\right|$. 
As shown in Eqn. 5 however, this correlation term leads to an enhanced electron crossfield mobility at instability saturation, which in the presence of electron-neutral collisions, reduces to:

$$
\mu_{\mathrm{eff}}^{s a t}=\frac{\frac{1}{m_{e} \nu_{m}}}{1+\frac{\omega_{c e}^{2}}{\nu_{m}^{2}}}\left[|q|+\frac{\omega_{c e}}{\nu_{m}} \frac{\left|\mathbf{R}_{e i}\right|}{n_{e} E_{0}}\right]
$$

Substituting Eqn. 9 into Eqn. 10, and simplifying then yields:

$$
\mu_{\mathrm{eff}}^{s a t}=\frac{\frac{|q|}{m_{e} \nu_{m}}}{1+\frac{\omega_{c e}^{2}}{\nu_{m}^{2}}}\left[1+\frac{|q|}{m_{e} \nu_{m}} \frac{B_{0}}{E_{0}} \frac{v_{z i} T_{e}}{4 \sqrt{6} c_{s} L_{z}}\right]
$$

By then using the known electron temperature and ion exit velocity in the simulations, Eqn. 11 can be used to predict the enhanced electron cross-field mobility. Interestingly, for the present simulation geometry, Eqn. 11 is independent of the plasma density, and depends only on the applied simulation conditions, electron-neutral collision frequency, and the electron temperature. Also, for $\nu_{m} \mapsto 0$, Eqn. 11 reduces to

$$
\mu_{\mathrm{eff}}^{\text {sat }}\left(\nu_{m} \mapsto 0\right) \approx \frac{1}{4 \sqrt{6}} \frac{v_{z i} T_{e}}{c_{s} B_{0} E_{0} L_{z}}
$$

As described by the kinetic theory in [37] the instability wavelength, $\lambda$, is given by:

$$
\lambda=2 \pi \lambda_{D e} \sqrt{2}
$$

where the Debye length, $\lambda_{D e}$, is defined as $\lambda_{D e}=\sqrt{\left(\epsilon_{0} T_{e}\right) /(|q| n)}$. Furthermore the frequency, $f$, of the instability is estimated using:

$$
f=\frac{\omega_{p i}}{2 \pi \sqrt{3}}
$$

With the ion plasma frequency defined as $\omega_{p i}=\sqrt{\left(n q^{2}\right) /\left(\epsilon_{0} m_{i}\right)}$. Finally the phase velocity, $v_{p h}$, is given by:

$$
v_{p h}=\sqrt{2 / 3} c_{s}
$$

We can now compare the results of the 2D PIC simulations with this theory. In order to study the impact of the plasma density on the instability behavior and electron transport, two further simulations were made using modified plasma densities of: $n=n_{0} / 4=0.75 \times 10^{17} \mathrm{~m}^{-3}$ and $n=4 n_{0}=12 \times 10^{17} \mathrm{~m}^{-3}$. These different simulations, together with the original at a density of $n_{0}$, will be referred to as " $n_{0}$ ", " $4 n_{0}$ " and 


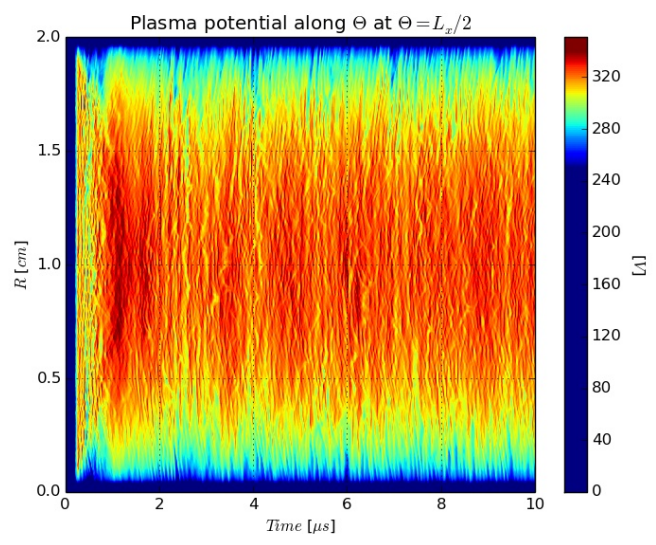

(a)

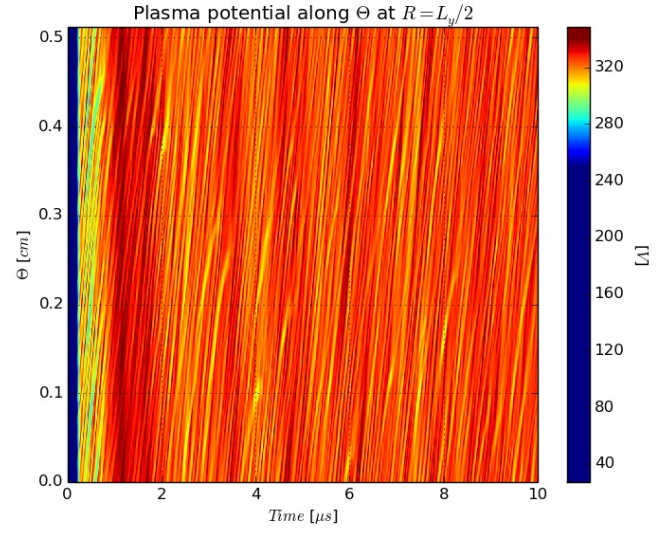

(b)

Figure 8: Time evolution plots of the plasma potential taken from $1 \mathrm{D}$ cuts of the 2D $(O x-O y)$ domain: (a) taken along $O y$ (or $r)$, at $x=L_{x} / 2\left(\right.$ or $\left.\theta=L_{\theta} / 2\right)$, (b) taken along $O x$ ( or $\theta)$, at $y=L_{y} / 2\left(r=L_{r} / 2\right)$. Collisions are enabled with parameters from Table 1, but the plasma density is increased to $4 n_{0}=12 \times 10^{17} \mathrm{~m}^{-3}$. The color-bar is rescaled in order to highlight the instability behavior.

" $n_{0} / 4 "$. Finally, all three simulations were done with electron-neutral collisions as well as ion-neutral collisions enabled. The results concerning the $n_{0}$ run can already be seen in Figure 7b, as well as in Figures 5a and 5b. The time evolution of the plasma potential along $O y$ and $O x$ for " $4 n_{0}$ " are shown in Figures $8 \mathrm{a}$ and $8 \mathrm{~b}$, while Figures $9 \mathrm{a}$ and $9 \mathrm{~b}$ show the results for " $n_{0} / 4$ ".

The first observation from these figures is the lower growth time of the instability for the higher density case. By contrast, the growth time is higher for the lower density case. This is consistent with the kinetic theory in [37], where the growth time, $\tau_{g}$, can be expressed in Eqn. 16:

$$
\tau_{g}=\frac{1}{2 \gamma_{\max }}
$$

with:

$$
\gamma_{\max }=\sqrt{\frac{\pi m_{e}}{54 m_{i}}} \frac{v_{d e}}{\lambda_{D e}}
$$

where $m_{e}$ is the electron mass, and $v_{d e}$ is the azimuthal electron drift velocity. Indeed if $n$ increases, $\lambda_{D e}$ decreases, leading to a higher growth rate $\gamma_{\max }$ and thus a lower growth time. Secondly, if the frequency and wavelength of the instability depends on the plasma density, we can observe that the phase velocity (directly measured in the time plots taken along $O x$ at $y=L_{y} / 2$ by using the slope of the instability propagation) is almost constant. These measurements are again consistent with the kinetic theory 


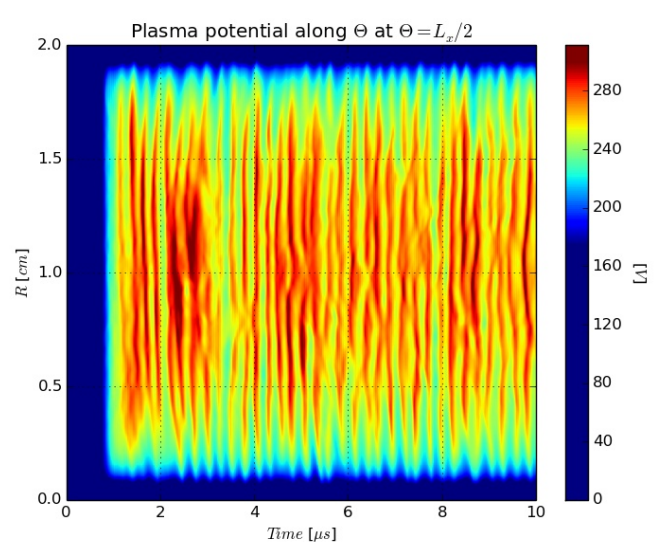

(a)

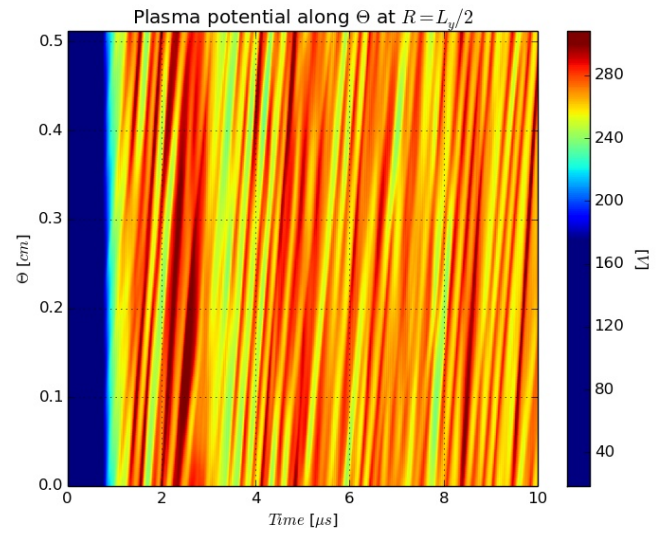

(b)

Figure 9: Time evolution plots of the plasma potential taken from 1D cuts of the 2D $(O x-O y)$ domain: (a) taken along $O y$ (or $r$ ), at $x=L_{x} / 2$ (or $\theta=L_{\theta} / 2$ ), (b) profile taken along $O x$ (or $\theta$ ), at $y=L_{y} / 2$ (or $\left.r=L_{r} / 2\right)$. Collisions are enabled with parameters from Table 1 , but the plasma density is reduced to $n_{0} / 4=0.75 \times 10^{17} \mathrm{~m}^{-3}$. The color-bar is rescaled in order to highlight the instability behavior.

in [37]. Comparisons between the results from the PIC simulations and the theoretical predictions are summarized for all three simulations in Table 2.

Measurements presented in Table 2 were done using the measured value of $T_{e}=$ $53[\mathrm{eV}]$. This value is taken from the mean value of the electron energy, $\epsilon_{e}$, by using the relation: $T_{e}=2 / 3 \epsilon_{e}$. Since this value is measured from the temporal plots of the energy as in Figure $3 \mathrm{~b}$, the uncertainty can be estimated from the plots to be $\approx \pm 5[\mathrm{eV}]$. This uncertainty is then echoed in $T_{e}$, and therefore also in the subsequent estimates. The oscillation amplitudes are measured directly from the time evolution plots from 1D cuts in the 2D domain. An example of such a plot is shown in Figures 5b, 8b, and $9 \mathrm{~b}$. These figures are used as well to measure the phase velocity, frequency and wavelength of the instability. Given the measurement method, uncertainty for $v_{p h}$ is in a range of $\approx \pm 0.5\left[10^{3} \mathrm{~ms}^{-1}\right]$, while being $\approx \pm 0.5[\mathrm{MHz}]$ for the frequency, and $\approx \pm 0.1[\mathrm{~mm}]$ for the wavelength.

The kinetic theory developed in [37] gives analytical expressions to describe the amplitude of the oscillations of the instability at saturation. Simulation values are measured by calculating the standard deviation at saturation of the oscillations, multiplied by $\sqrt{2}$. The theory then predicts a value of $\left|\delta \widetilde{n}_{e}\right| / n_{e} \approx|\delta \widetilde{\Phi}| / T_{e}=1 / 3$. As can be seen in Table 2 , where $\left|\delta \widetilde{n}_{e}\right| / n_{e} \approx 0.12-0.2$, and $|\delta \widetilde{\Phi}| / T_{e} \approx 0.155-0.32$, the predicted values are in reasonable agreement, but tend to over-predict the instability amplitude. By comparison, the instability wavelength, frequency, and phase velocity, are in quite good agreement with the values obtained by the 2D PIC simulations.

As shown in Section 6 above, for the present geometry, the electron cross-field mobility when the instability is saturated is independent of the plasma density. This 
Table 2: Comparison between physical values measured from the simulation and predictions from the kinetic theory about the instability characteristics.

\begin{tabular}{cccc}
\hline \multicolumn{4}{l}{ Measured values } \\
\hline Case & $\lambda[\mathrm{mm}]($ Eqn. 13$)$ & $f[\mathrm{MHz}]($ Eqn. 14$)$ & $v_{p h}\left[10^{3} \mathrm{~ms}^{-1}\right]$ (Eqn. 15) \\
\hline$n_{0} / 4$ & 2.0 & 2.5 & 5.0 \\
$n_{0}$ & 1.0 & 5.0 & 5.0 \\
$4 n_{0}$ & 0.7 & 10.0 & 5.0 \\
\hline Case & $\left|\delta \widetilde{n}_{e}\right| / n_{e}[\%]$ & $|\delta \widetilde{\Phi}| / T_{e}[\%]$ & \\
\hline$n_{0} / 4$ & 20 & 32 & \\
$n_{0}$ & 17 & 25 & \\
$4 n_{0}$ & 12 & 15.5 & \\
\hline Analytical values & & 5.011 \\
\hline Case & $\lambda[\mathrm{mm}]$ & $f[\mathrm{MHz}]$ & 5.011 \\
\hline$n_{0} / 4$ & 1.7 & 2.9 & 5.011 \\
$n_{0}$ & 0.8 & 5.8 & \\
$4 n_{0}$ & 0.4 & 11.6 & \\
\hline Case & $\left|\delta \widetilde{n}_{e}\right| / n_{e}[\%]$ & $|\delta \tilde{\Phi}| / T_{e}[\%]$ & \\
\hline$n_{0} / 4$ & 33 & 33 & \\
$n_{0}$ & 33 & 33 & \\
$4 n_{0}$ & 33 & 33 &
\end{tabular}

allows us to predict the mobility from the simulation parameters, using the calculated values of $\nu_{m}$ and $T_{e}$. The results from the simulation and Eqn. 11 are presented in Table 3. Measurement is done by temporally averaging $\mu_{\text {pic }}$ as well as $\mu_{\text {eff }}$ from $t=5.0 \mathrm{~ms}$ to $t=10.0 \mathrm{~ms}$. Despite measurement uncertainties, the values are in quite good agreement.

In order to compare these results with the classical mobility definition as given by Eqn. 4, $\mu_{\text {classical }}$ is estimated in the same conditions. As expected the result is about 50 times lower than that found in the PIC, $\mu_{\text {pic }}$, and that predicted from the kinetic theory, $\mu_{\mathrm{eff}}$.

\section{Discussion and Conclusions}

In the sections above we have presented the results from a simplified $(O x-O y)$ (or $r-\theta)$ PIC simulation where enhanced electron cross-field transport has been observed, as well as a strong instability in the azimuthal direction. It has been seen that the instability and the enhanced electron transport are strongly associated, leading to an effective cross-field electron mobility many times larger than that from classical electron/neutral collisions. 
Table 3: Comparison between physical values measured from the simulation and predictions from the kinetic theory for the electron cross-field mobility.

\begin{tabular}{ccccc}
\hline Case & \multicolumn{2}{c}{ Measured values } & \multicolumn{2}{c}{ Analytical values } \\
\hline$\left[\mathrm{m}^{2} \mathrm{~V}^{-1} \mathrm{~s}^{-1}\right]$ & $\mu_{\text {pic }}($ Eqn. 3) & $\mu_{\text {eff }}$ (Eqn. 5) & $\mu_{\text {eff }}^{\text {sat }}$ (Eqn. 11) & $\mu_{\text {classical }}$ (Eqn. 4) \\
\hline$n_{0} / 4$ & 6.0 & 5.9 & 4.23 & 0.111 \\
$n_{0}$ & 5.8 & 5.6 & 4.23 & 0.111 \\
$4 n_{0}$ & 6.1 & 6.0 & 4.23 & 0.111 \\
\hline
\end{tabular}

This instability acts as an important momentum loss mechanism in addition to standard electron-neutral collisions. Although the instability, and consequently the momentum loss, occurs in the azimuthal direction, the presence of the magnetic field couples this to the axial direction. Complimenting previous 1D PIC results, these 2D PIC simulations highlight that this process is mostly independent of electron-neutral collisions, and does not require electron-wall collisions or secondary electron emission. Confirming the kinetic theory developed in [36] and [37], this study verifies that the enhanced cross-field electron mobility depends on an electron density-electric field correlation term, which represents an electron-ion friction force that is strongly enhanced by the instability.

Although numerous studies have highlighted the role of electron-wall collisions and secondary electron emission on the electron transport $[3,11,12,22]$, the results presented above confirm the 1D PIC studies [36], as well as the 2D PIC simulations from [15, 34], all of which did not include any secondary electron emission, and hence which suggests that wall effects probably play only an auxiliary role. It is of course experimentally observed that changes of a real HET wall materials modify the electron transport. However, since such a change would also modify the particle distribution functions and hence the plasma/discharge properties, and since the instability is a function of these properties, any modifications to the wall materials can be expected to affect the electron transport. The instability however is still the primary cause for the anomalous transport.

Since the focus of the present work has been to concentrate on the basics physics, a simplified model has been used, and it is important to highlight and discuss some limitations linked to this approach. Firstly, the obvious limitations are that: (1) the walls are metallic and grounded, (2) no secondary electron emission is integrated in the model. Both of these points are expected to alter the equilibrium values reached by the simulation (such as the plasma potential), but not necessarily the overall conclusions on electron transport. Adding in a model to account for secondary electron emission, as well as dielectric walls, would let us more realistically simulate a real device. A less obvious limitation of the present simulations is that Poisson's equation is only solved in the $r-\theta$ plane, and so the wavenumber of any fluctuations along $O z$ are zero. This implies that convection of the instability away from the simulation plane is not correctly modeled. We have tried to account for this by using a finite axial length and removing particles which cross the boundaries in this direction, but this still only represents an 
approximation. Efforts are under way to improve the present code and remove some of the above limitations. Nevertheless, these simplified simulations still give useful insights on the basic physical mechanisms governing the instability formation and the associated electron transport.

\section{Acknowledgements}

The authors would like to thank Francois Pechereau, Abdoul Wahid C. Mainassara, Antoine Tavant, Loic Nicolas, and Stephan Zurbach for their numerous and useful discussions as well as for their help and support in the development of the LPPic2D simulation code. V.C. acknowledges financial and technical support from a Safran Aircraft Engines (former SNECMA) doctoral research award, as well as from the Association Nationale de la Recherche et de la Technologie (ANRT) as part of a CIFRE convention. T.L. acknowledges financial support from a CNES postdoctoral research award. Z.B. acknowledges support from the Czech Science Foundation research project 15-04023S and project LO1411 (NPU I) funded by the Ministry of Education, Youth and Sports of the Czech Republic.

\section{Appendix}

\section{D capacitive model and verifications}

To test and verify the results of the LPPic2D code, a 2D capacitive model was used in order to compare with the results obtained in [43]. Although this capacitively coupled plasma (CCP) benchmark may, at first sight, appear to model a system far from the plasma discharge conditions encountered in HETs, these CCP benchmarks are some of the few complete benchmarks available for low-pressure PIC codes, and allow us to test most of the subroutines and functions to be used in the final code. In [43], four benchmarks were presented to model a Helium CCP discharge for different simulation conditions. In each case, five independent 1D PIC codes were compared and shown to give statistically indistinguishable results when using identical simulation conditions and numerical parameters. In this work we have chosen the first benchmark case as it uses the lowest pressure condition, and is thus the "most similar" to a HET.

Figure 10 shows the geometry of the 2D-3V capacitive system described. In this system the upper and lower walls are metallic, fully absorbing, and either grounded (at $y=0$ ) or powered (at $y=y_{\max }$ ) with a sinusoidal potential, $V(t)=V_{0} \sin (2 \pi f t$ ), at a frequency $f$ and an amplitude $V_{0}$. This oscillating potential, coupled with the possibility for charged particles to have collisions with neutrals (in particular ionization), allows the simulation to be self-consistent and to reach a dynamic steady state after many time steps.

Numerous tests were performed to qualify each of the subroutines used in the code, but to test the entire code, a global benchmark was made to compare with the 1D results obtained in [43]. To achieve this, the upper and lower metallic walls are closed, 


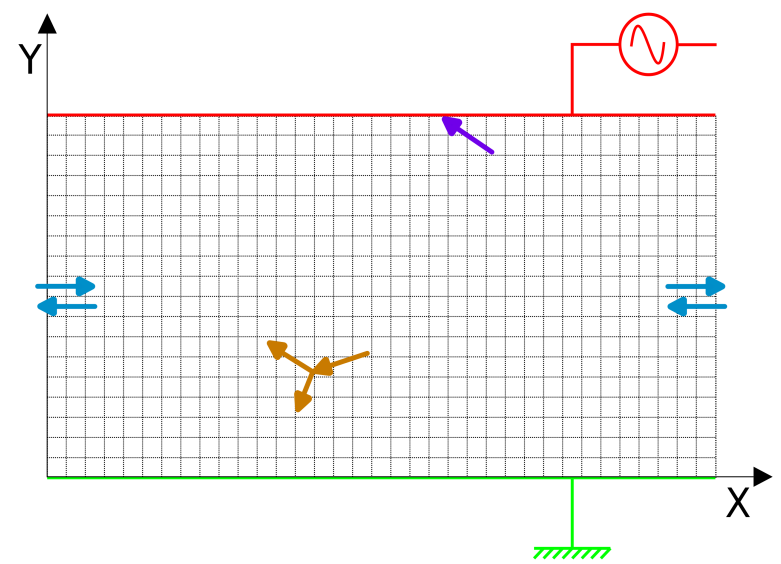

Figure 10: 2D capacitive model. Fully absorbing metallic walls (violet) along $O x$, grounded at $y=0$ (green), and powered with a sinusoidal potential at $y=y_{\max }$ (red). The transverse walls use periodic boundary conditions (blue), and simulations include ionization, excitation and elastic collisions (brown).

while the transverse walls use periodic boundary conditions. Thus the $2 \mathrm{D}$ system is essentially equivalent to a 1D CCP system.

Table 4: Standard operating and numerical parameters used in the 2D PIC simulations in order to compare the results with the 1D PIC benchmark results from [43]

\begin{tabular}{cc}
\hline Parameter & Value \\
\hline Gas & Helium \\
$L_{Y}[\mathrm{~cm}]$ & 6.7 \\
$L_{X}[\mathrm{~cm}]$ & 0.42 \\
$V_{0}[\mathrm{~V}]$ & 450 \\
$f[\mathrm{MHz}]$ & 13.56 \\
$n_{0}\left[\mathrm{~m}^{-3}\right]$ & $2.56 \times 10^{14}$ \\
$\Delta t[\mathrm{~s}]$ & $(400 f)^{-1}$ \\
$\Delta x=\Delta y[\mathrm{~m}]$ & $L_{Y} / 128=L_{X} / 8$ \\
$T_{e}[\mathrm{eV}]$ & 2.6 \\
$T_{i}[\mathrm{eV}]$ & 0.026 \\
$T_{n}[\mathrm{eV}]$ & 0.026 \\
$n_{g}\left[\mathrm{~m}{ }^{-3}\right]$ & $9.64 \times 10^{20}$ \\
$N[$ particles $]$ & 262144 \\
$N G[$ gridpoints $]$ & $128 \times 8$ \\
$N / N G[\mathrm{part} /$ cell $]$ & 256 \\
$N_{\text {steps }}[\Delta t]$ & 512000 \\
$N_{\text {average }}[\Delta t]$ & 12800
\end{tabular}

The simulation is initialized using parameters listed in Table 4 , where $n_{0}$ is the 
initial density, $T_{e}$ and $T_{i}$ are the initial electron and ion temperatures respectively, $T_{n}$ and $n_{g}$ are the neutral temperature and neutral density, $N$ is the number of particles in the system, and $N G$ is the number of grid points. Helium is used and both electrons and singly-charged Helium ions are modeled. Helium neutrals are considered as a homogeneous and constant background. Electron/neutral and ion/neutral collision processes are modeled. $N_{\text {steps }}$ is the number of time-steps modeled, and $N_{\text {average }}$ is the number of time-steps (with step size $\Delta t$ ) used to average the diagnostic output values. Taking a mean value along the $O x$ axis allows us to compare profiles along the $O y$ axis with the $1 \mathrm{D}$ results. Figures $11 \mathrm{a}$ and $11 \mathrm{~b}$ show the ion density and the ionization source term as a function of $y / L_{y}$ calculated with the LPPic2D code and from the $1 \mathrm{D}$ codes in [43]. It is important to note that the $1 \mathrm{D}$ results are smoother since they are obtained by averaging the results from the simulations made with the different codes in that work. Nevertheless the discrepancy observed between the results of the 2D LPPic2D code and of $1 \mathrm{D}$ PIC codes in [43] is small, which validates the results obtained with the LPPic2D code.

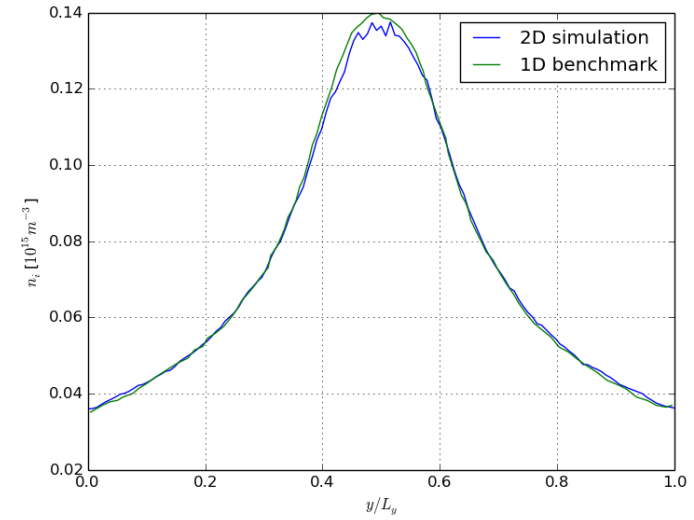

(a)

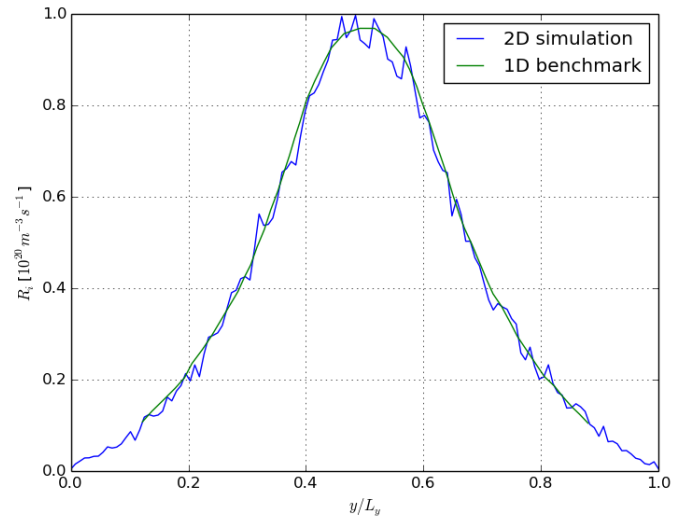

(b)

Figure 11: Comparison between the results from the LPPic2D code using the operating parameters from Table 4 (blue) and the 1D PIC benchmark results from [43] (green): (a) Ion density as a function of $y / L_{y}$, (b) Ionization source term as a function of $y / L_{y}$. 


\section{References}

[1] E. Y. Choueiri. A critical history of electric propulsion: The first 50 years (1906-1956). J. Propul. Power, 20(2):193-203, 2004.

[2] S. Mazouffre. Electric propulsion for satellites and spacecrafts: Established technologies and novel approaches. Plasma Sources Sci. Technol., 25(3), 2016.

[3] D. M. Goebel and I. Katz. Fundamentals of Electric Propulsion: Ion and Hall Thrusters. Wiley, 2008.

[4] R. Villain. Satellites to be built and launched by 2014, volume 18. Euroconsult Research Report, 2015.

[5] A. Bouchoule, A. Cadiou, A. Heron, M. Dudeck, and M. Lyszyk. An overview of the French research program on plasma thrusters for space applications. Contrib. Plasma Phys., 41(573), 2001.

[6] J. C. Adam, J. P. Boeuf, N. Dubuit, M. Dudeck, L. Garrigues, D. Gresillon, A. Heron, G. Hagelaar, V. Kulaev, N. Lemoine, S. Mazouffre, J. Perez-Luna, V. Pisarev, and S. Tsikata. Physics, simulation, and diagnostics of Hall effect thrusters. Plasma Phys. Control. Fusion, 24(124041), 2008.

[7] A. I. Morozov, V. V. Savelyev edited by B. B. Kadomtsev, and V. D. Shafranov. Reviews of Plasma Physics. Springer Science+Business Media, New-York, 2000.

[8] N. B. Meezan, W. A. Jr Hargus, and M. A. Cappelli. Anomalous electron mobility in a coaxial Hall discharge plasma. Phys. Rev., E 63, 026410, 63(2), 2001.

[9] L. Garrigues, J. Pérez-Luna, J. Lo, G. J. M. Hagelaar, J.P. Boeuf, and S. Mazouffre. Empirical electron cross-field mobility in a Hall effect thruster. Appl. Phys. Lett., 95(141501), 2009.

[10] I. Katz, I. G. Mikellides, B. A. Jorns, and A. L. Ortega. Hall2De simulations with an anomalous transport model based on the electron cyclotron drift instability. In IEPC-2015-402, 2015.

[11] I. D. Kaganovich, Y. Raitses, D. Sydorenko, and A. Smolyakov. Kinetic effects in a Hall thruster discharge. Phys. Plasmas, 14(057104), 2007.

[12] D. Sydorenko, A. Smolyakov, I. Kaganovitch, and Y. Raitses. Electron kinetic effects and beam related instabilities in Hall thrusters. Phys. Plasmas, 15(053506), 2008.

[13] F. Taccogna, S. Longo, M. Capitelli, and R. Schneider. Anomalous transport induced by sheath instability in Hall effect thrusters. Appl. Phys. Lett., 94(251502), 2009.

[14] M. Hirakawa. Electron transport mechanism in a Hall thruster. In IEPC-97-021, 1997.

[15] J. C. Adam, A. Héron, and G. Laval. Study of stationary plasma thrusters using two-dimensional fully kinetic simulations. Phys. Plasmas, 11(295), 2004.

[16] A. Ducrocq, J. C. Adam, A. Héron, and G. Laval. High-frequency electron drift instability in the cross-field configuration of Hall thrusters. Phys. Plasmas, 13(102111), 2006.

[17] A. Héron and J. C. Adam. Anomalous conductivity in Hall thrusters : Effects of the non-linear coupling of the electron-cyclotron drift instability with secondary electron emission of the walls. Phys. Plasmas, 20(082313), 2013.

[18] A. Morozova, Y. Esipchuk, A. Kapulkin, V. Nevrovskii, and V. Smirnov. Effect of the Magnetic Field on a Closed-Electron-Drift Accelerator. Sov. Phys.: Tech. Phys., 17(482), 1972.

[19] D. Escobar and E. Ahedo. Low frequency azimuthal stability of the ionization region of the Hall thruster discharge. Phys. Plasmas, 21(043505), 2014.

[20] W. Frias, A. I. Smolyakov, I. D. Kaganovitch, and Y. Raitses. Long wavelength gradient drift instability in Hall plasma devices. I. Fluid theory. Phys. Plasmas, 19(072112), 2012.

[21] W. Frias, A. I. Smolyakov, and I. D. Kaganovitch. Long wavelength gradient drift instability in Hall plasma devices. II. Applications. Phys. Plasmas, 20(052108), 2012.

[22] N. Gascon, M. Dudeck, and S. Barral. Wall material effects in stationary plasma thrusters. I. Parametric studies of an SPT-100. Phys. Plasmas, 10(4123), 2003.

[23] C. Boniface, L. Garrigues, G. J. M. Hagelaar, J. P. Boeuf, D. Gawron, and S. Mazouffre. Anomalous cross-field electron transport in a Hall thruster. Appl. Phys. Lett., 89(161503), 
2006.

[24] G. J. M. Hagelaar, J. Bareilles, L. Garrigues, and J. P. Boeuf. Role of anomalous electron transport in a stationary plasma thruster simulation. J. Appl. Phys., 93(67), 2003.

[25] N. B. Meezan and M. A. Capelli. Kinetic Study of Wall Collisions in a Coaxial Hall Discharge. Phys. Rev., E 66(036401), 2002.

[26] F. I. Parra, E. Ahedo, J. M. Fife, and M. Martinez-Sanchez. A two-dimensional hybrid model of the hall thruster discharge. J. Appl. Phys., 100(023304), 2006.

[27] A. N. Smirnov, Y. Raitses, and N. J. Fisch. Electron cross-field transport in a miniaturized cylindrical Hall thruster. IEEE Trans. Plasma Sci., 34(132), 2006.

[28] S. Yoshikawa and D. J. Rose. Anomalous Diffusion of a Plasma across a Magnetic Field. Phys. Fluids, 5(334), 1962.

[29] G. S. Janes and R.S. Lowder. Anomalous Electron Diffusion and Ion Acceleration in a LowDensity Plasma. Phys. Fluids, 9(115), 1966.

[30] E. Y. Choueiri. Fundamental difference between the two Hall thruster variants. Phys. Plasmas, 8(1411):5025-5033, 2001.

[31] A. W. Smith and M. A. Cappelli. Time and space-correlated plasma potential measurements in the near field of a coaxial Hall plasma discharge. Phys. Plasmas, 16(073504), 2009.

[32] M. K. Scharfe, N. Gascon, M. A. Cappelli, and E. Fernandez. Comparison of hybrid Hall thruster model to experimental measurements. Phys. Plasmas, 13(083505), 2006.

[33] J. Cavalier, N. Lemoine, G. Bonhomme, S. Tsikata, C. Honore, and D. Gresillon. Hall thruster plasma fluctuations identified as the E x B electron drift instability : Modeling and fitting on experimental data. Phys. Plasmas, 20(082107), 2013.

[34] P. Coche and L. Garrigues. A two-dimensional (azimuthal-axial) Particle-In-Cell model of a Hall thruster. Phys. Plasmas, 21(023503), 2014.

[35] J. P. Boeuf. Rotating structures in low temperature magnetized plasmas - insight from particle simulations. Front. Phys., 2(74), 2014.

[36] T. Lafleur, S. D. Baalrud, and P. Chabert. Theory for the anomalous electron transport in Hall effect thrusters: I Insights from particle-in-cell simulations. Phys. Plasmas, 23(053502), 2016.

[37] T. Lafleur, S. D. Baalrud, and P. Chabert. Theory for the anomalous electron transport in Hall effect thrusters: II. Kinetic model. Phys. Plasmas, 23(053503), 2016.

[38] C. K. Birdsall and A. B. Langdon. Plasma Physics via Computer Simulation. McGraw-Hill, New-York, 1985.

[39] I. Dupays, M. Fle, J. Gaidamour, D. Girou, P.F. Lavallée, D. Lecas, and P. Wautelet. Message Passing Interface (MPI), volume 4.7.3. IDRIS, 2015.

[40] J. P. Boris. Relativistic plasma simulation-optimization of a hybrid code. In Proceedings of the 4 th Conference on Numerical Simulation of Plasmas. Naval Res. Lab., pages 3-67, Washington DC, 1970 .

[41] V. Vahedi and M. Surendra. A Monte Carlo collision model for the particle-in-cell method: applications to argon and oxygen discharges. Comp. Phys. Commun., 87(179), 1995.

[42] A. V. Phelps. Compilation of atomic and molecular data. 2005.

[43] M. M. Turner, A. Derzsi, Z. Donkó, D. Eremin, S. J. Kelly, T. Lafleur, and T. Mussenbrock. Simulation benchmarks for low-pressure plasmas: Capacitive discharges. Phys. Plasmas, 20(013507), 2013.

[44] R. D. Falgout, J. E. Jones, and U. Meier Yang. The design and implementation of hypre, a library of parallel high performance preconditioners, volume 51 of Lecture Notes in Computational Science and Engineering. Springer, 2006.

[45] The HDF Group. Hierarchical Data Format, version 5, 1997-2016.

[46] R. L. Dewar. Saturation of kinetic plasma instabilities by particle trapping. Phys. Fluids, 16(431435), 1973.

[47] S. Mazouffre. Laser-Induced fluorescence diagnostics of the cross-field discharge of Hall Thrusters. Plasma Sources Sci. Technol., 22(013001), 2012. 
[48] T. Lafleur, S. D. Baalrud, and P. Chabert. Characteristics and transport effects of the electron drift instability in Hall-effect thrusters. Plasma Sources Sci. Technol., submitted.

[49] T. Lafleur, P. Chabert, and J.P.Booth. Electron heating in capacitively coupled plasmas revisited. Plasma Sources Sci. Technol., 23(035010), 2014. 